\title{
Quelques additions au Glossaire de Pierrecourt.
}

\section{Formes correspondant à certains mots obscurs du patois de Pierrecourt (Haute-Saône).}

Pendant mes vacances de 1913 je me suis proposé de chercher, dans des patois apparentés à celui de Pierrecourt, des formes correspondant aux mots dont je n'ai pu donner d'étymologie plausible dans mon Glossaire du patois de Pierrecourt.1 J'ai interrogé des personnes appartenant aux treize localités suivantes: Larret, SaintAndoche, tous deux du canton de Champlitte (Haute-Saône) et situés le premier à $4 \mathrm{~km}$, le second à $8 \mathrm{~km}$ de Pierrecourt; Chameroy, canton d'Auberive, Serqueux, canton de Bourbonne-lesBains, Voisey entre Bourbonne et Vitrey, tous trois situés dans le sud de la Haute-Marne; - Nonville, canton de Monthureux-surSaône, Val d'Ajol, tous deux situés dans le sud des Vosges; Magny-lès-Jussey, Provenchère près Faverney, Menoux, canton d'Amance, Froideconche près Luxeuil, tous quatre situés dans le département de la Haute-Saône, les trois premiers dans l'arrondissement de Vesoul; - Delle (Haut-Rhin); - Rougemont (Doubs), chef-lieu de canton. Comme Larret et Saint-Andoche ont en général les mêmes formes que Pierrecourt, je mentionnerai ces deux villages seulement lorsqu'ils offrent une prononciation spéciale. J'ai trouvé aussi quelques indications pour Plancher-les-Mines (nordest de la Haute-Saône) dans l'Essai d'un vocabulaire étymologique du patois de Plancher-les-Mines par le Dr. Poulet (1878), et pour le Doubs dans le Vocabulaire étymologique des provincialismes du Doubs par Ch. Beauquier (1881).

Dans les listes suivantes je mentionne, sauf pour Larret et Saint-Andoche, toutes les formes que j'ai recueillies dans les diverses localités. Quand je passe sous silence certains patois, cela veut dire seulement que je n'ai pas de renseignements à donner. Cela

1 51. Beiheft zur Zeitschr. f. roman. Phil. 
ne signifie aucunement que des formes correspondantes n'existent pas dans ces patois. Dans chaque localité j'ai interrogé sur tous les mots de ma liste. Mais bien des hasards ont pu m'empêcher de recueillir toutes les formes correspondantes qui existent en réalité. Pour établir la non-existence d'une forme dans un patois, il faudrait des enquêtes étendues que je n'ai pu faire.

Voici les mots de ma liste pour lesquels je n'ai rencontré aucune forme correspondante dans les localités citées, sauf Larret

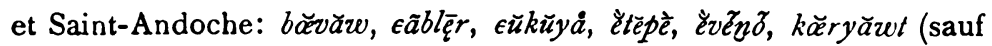
Rougemont kăayrot, cf. Mandeure dans le Doubs: cœuillerate, selon Vautherin Patois de Chatenois), krāazbăw, mëgè, olizgên, răbăs, rnăzẹ̄l,

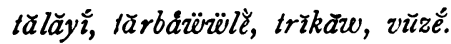

Quant aux autres mots, je range les formes des divers patois dans l'ordre qui vient d'être suivi pour l'énumération des localités, de telle sorte que cet ordre va en gros de l'ouest à l'est, les formes relevées à Rougemont ou chez $M$. Beauquier ou ailleurs étant ajoutées en appendice. La forme placée en tête de chaque article est toujours celle de Pierrecourt. Les autres formes ont, sauf indication contraire, le même sens que celle de Pierrecourt.

Les correspondances relevées m'ont rarement amené à confirmer ou infirmer une hypothèse étymologique ou conduit à une solution étymologique sûre. Toutefois, en dehors de cette question de l'origine des mots, il y a celle de l'extension géographique actuelle des mots, et à cet égard j'espère que mon enquête ne sera pas tout à fait dépourvue d'intérêt.

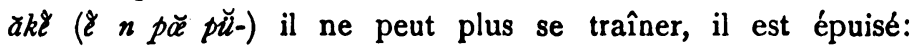
Chameroy $r k e$, , Voisey $a k \grave{\psi}$.

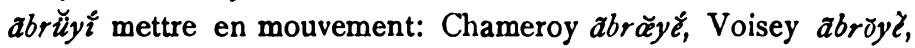

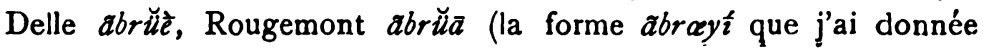
dans le (Glossaire est un barbarisme), Beauquier embruer. Identique d̀ fr. embrayer.

$\tilde{a} \bar{a} \bar{a} m \breve{~ e n t r e p r e n d r e ~ t r o p ~ d e ~ b e s o g n e ~ a ̀ ~ l a ~ f o i s ~ e t ~ l a ~ m a l ~ f a i r e: ~}$ Chameroy $\bar{a} \bar{a} \bar{a} m \tilde{t}$ barbouiller de choses gluantes, poisseuses; Serqueux et Voisey agāmĕ faire deux ou trois ouvrages à la fois. Le sens de ce mot à Chameroy est exactement celui d'ägămŭr faut-il en conclure qu'agamŭrĕ serait un dérivé d'agāmĕ? Pour le sens on pourrait comparer: bousiller, gâcher de l'ouvrage.

agž̈ communiquer une maladie contagieuse: Serqueux åję, Nonville $b_{j} \ddot{\text {, }}$, Rougemont mal qui $a g h \hat{h}$, Beauquier s' enguer se dit d'une maladie qui s'aggrave, dolois (arr de St. Mâlo) angoué en- 
dolori, en parlant d'un organe fatigué par un grand travail ( $L e$ parler dolois par Ch. Lecomte 1910).

$\tilde{a} l y z$ (f.) sotte, maladroite: même forme et même sens à Serqueux, Voisey, Nonville, Menoux, Froideconche.

atărló $(\mathrm{m}$.) courroie qui réunit les deux parties d'un fléau: Provenchère et Rougemont atrălë; Chatenois enterli (Pat. de Chatenois par Vautherin).

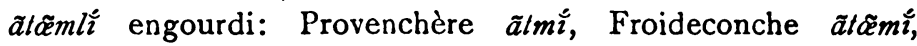
Plancher entemit, Rougemont ătmĭ, Beauquier entemis. Pour l'étymologie v. A. Thomas Romania juillet 1913 p. 394 et suiv.

ava ălè s'enflammer (se dit d'une plaie qui s'enflamme): Provenchère et Rougemont ãvāla s'enflammer (en parlant d'un feu, d'un incendie, non d'une plaie), Beauquier s'envoiler (en parlant du feu), Chatenois onvielai être en flammes (envélâ Auxel).

$b \gamma k$ (f.) grosses noix, avec lesquelles les enfants jouent de préférence: Beauquier boque grosse bille (jeu).

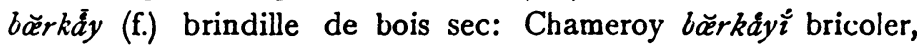

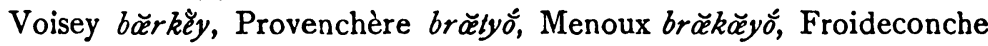
brăkžyŏ, Rougemont brăkyys, Beauquier brequillon. Ces mots sont sans doute dérivés de allem. brechen, v. Meyer-Lübke, Roman. etym. Wb. art. brikan, 3 .

$b z \bar{a} y \dot{y}$, verbe qui se dit des vaches, lorsque, piquées des mouches, elles se sauvent en levant la queue: Chameroy bziyč, Serqueux

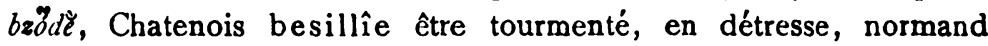
beser cité par Ch. Lecomte (Parler dolois, art. moucher).

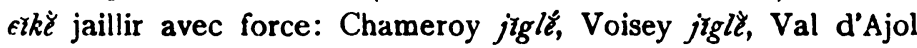
jikla, Rougemont teikyā; cf. fr. mod. gicler.

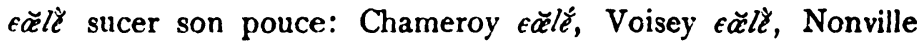

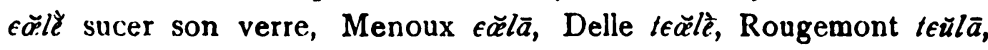
Beauquier chuler, dolois chuler boire (surtout fréqueminent, $v$. Parler dolois).

drālě trotter à droite et à gauche: Chameroy drălêt courir très vite. Parent de troler?

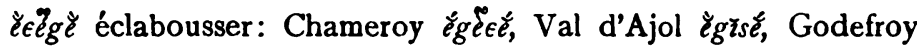
esguissouere éclissoire, Chatenois éguisser éclabousser, îedyisse clifoire.

๕̆gẽn (f.) alise: Rougemont ไ̆grot.

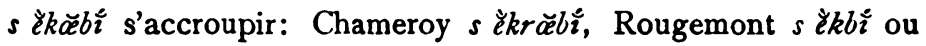

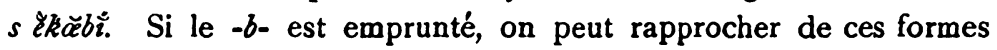
le lorrain akové v. Roman. etym. Wb. art. cubāre. 
fyåzö $\epsilon$ s'emploie à Pierrecourt non seulement dans l'expression făr fydz̋̈e (v. Glossaire), mais encore comme adjectif, surtout en parlant de l'étoffe pour dire qu'elle ne se tient pas raide: Saint-

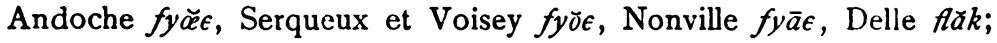
partout employé comme adjectif; Godefroy flac mou. Toutes ces formes semblent remonter à flaccum -am, v. Roman. etym. Wb. art. flaccus. besogne.

flyo̊b lancer avec force: Voisey vyőbi aller vite et fort en

fărt

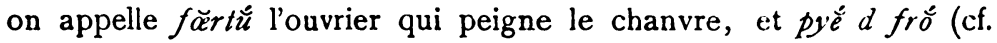
au glossaire de Pierrecourt pyĕ $d$ frăw) le chanvre le plus grossier; à Rougemont fértā signifie presser le chanvre sur un certain instrument pour en enlever l'écorce rugueuse: métathèse de fr. frotter, cf. vénitien fraton polissoir, v. Roman. elym. Wb. art. *frictāre.

găsŭyı̆ se dit des enfants qui salissent l'eau en la remuant: Chameroy gāeŭyé, Serqueux găsŭyĕ, Provenchère et Menoux gãvŭy Delle găzwěyt, Rougemont gzvwăyt, Beauquier gavouiller, Chatenois gavouëyîe barboter dans l'eau sale. La forme gäєŭyé semble dérivée de v. h. allem. waschan, d'où fr. gouache. Voir găwwjï.

$s$ găzewoǰ s'emplir d'eau la chaussure en marchant dans une flaque d'eau: Voisey $s g \bar{a} \epsilon \check{e}$, Beauquier gauger se mouiller les pieds (mains). ga $\bar{a} \in \ddot{l}$ fait penser au v. h. allem. waschan; les autres formes sont peut-être le résultat de contaminations.

gd̃ürümĕ se dit surtout des aliments qu'on a laissés trop longtemps sur le feu: Chameroy gämĕ, Rougemont gümā faire tremper les légumes de telle sorte qu'ils augmentent de poids, Chatenois goumầ, être en activité latente, en parlant du feu, d'une maladie, etc., Beauquier goumer ou gommer cuire à petit feu. Toutes formes empruntées de cauma (v. Glossaire), ainsi que le prouvent le niçois caumar, qui se dit des mets qui, en se refroidissant, perdent de leur fraîcheur et de leur saveur naturelles (v. Calvino, Nouveau dictionn. nigois-français 1905) - et le dolois chaumir brûler par la sècheresse (Le parler dolois par Ch. Lecomte 1910).

gërōy (f.) jeune fille d'allures trop libres: Chameroy gĕ̀rōy, Voisey gădūy, Nonville gărūy.

$g \bar{o} y$ (f.) sachet de linge pour laver la vaisselle: Chameroy gōy femme qui s'habille mal (= en chiffons), Serqueux gōy, Voisey gwoyy, Nonville gōy chiffon, Menoux main de gwăy c-d̀-d. main qui n'a 
pas plus d'énergie qu'un chiffon, Delle găy chiffon, Beauquier goille ou gaille chiffon, Chatenois gaille guenille.

gryarwt (f.) bourse en forme de sachet: Chameroy gōyzt, Serqueux et Voisey et Nonville gøyot, Menoux gwăyat, Froideconche gwăyðt, Val d'Ajol gwð̌yăt. A Voisey gðyðt signifie aussi $g \bar{o} y$.

grěvõnĕ se dit des poules qui grattent le sol avec leurs pattes:

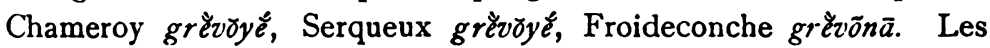

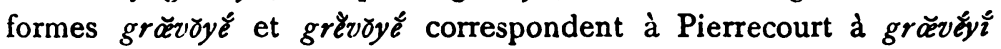
démanger, picoter. Dérivés de v. h. allem. graban.

jäs (donner les -), f. pl., agacement des dents qu'on éprouve en mangeant des fruits non mûrs: Larret et Saint-Andoche $\varepsilon_{\epsilon}$, Rougemont djěs, Beauquier jes et gences. - Serqueux dents $\bar{a} z \hat{z}$ (adj.), Nonville ăsyě́ les dents (verbe), Val d'Ajol dents āsî́ (adj.) continuent $v$. fr. a acier $>{ }^{*}$ adaciāre et probablement n'ont rien à faire avec $j$ ăs. De ce dernier M. G. Bertoni propose de rapprocher le limousin janzi agacement (communiqué par M. Chabaneau .en 1910), et le provençal genzic (v. G. Bertoni, Gesellschaft für roman. Literatur vol. III, p. 65).

lărjăwt (f.) laitron des champs: Chameroy lărjăt, Serqueux et Voisey lărjot, Provenchère larjăt, Menoux lërjăt, Froideconche lërjot. kălizeăwt (f.) cime d'un arbre, d'un épi: Chameroy, Serqueux,

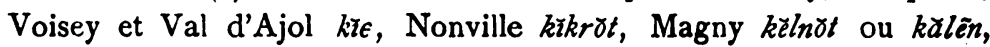
Provenchère et Menoux kaleñ.

kălwè̌ (m.) sorte de poire: Serqueux kălwě́, Voisey kălwet, Nonville kălwé.

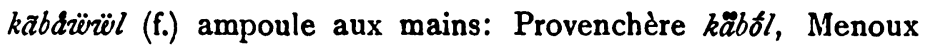
$k a \tilde{b} b$ bl cal, durillon. A Nice cai signifie aussi cal et ampcule (v.

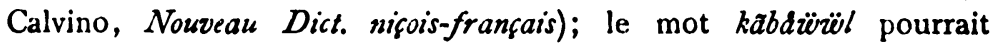
donc être une contamination de cal + ampoule altéré d'après boule.

$k a ̋ k z b w e \bar{l} l$ (f.): Chameroy, Serqueux, Voisey kăkžbwĕl, Nonville $k a ̈ k z b b l$, Froideconche kikäból.

$k \delta r p \check{e}(\mathrm{~m}$.$) planche de légumes: Serqueux korpé. - Godefroy$ coupelle mesure de terre?

kótb (m.) côte (de chou, etc.): id. à Voisey, Provenchère, Froideconche, Rougemont, et chez Beauquier.

krătăw (m.) nuque et creux sur la nuque: Larret krðtăw, Saint-Andoche krotó, Chameroy krätnü. Ces formes dérivent peutêtre de crypta, qui a donné en v. fr. crote, croton grotte, croté creux. 
lốn ou lōnẽ très lent (se dit d'une personne): Larret lăwwn, Rougemont lünŭ, Chatenois lodi ou lōdîe fainéant qui mendie.

lăgॄ̋ (m.) purin: Chameroy lăgĕ toute grande flaque d'un liquide sale, Serqueux et Voisey lojë, Magny lojë, Provenchère et Menoux $l j \ddot{t}$, Froideconche $l \delta j \ddot{z}$, Plancher $\log \bar{i}$.

lūzz (f.) maladie épidémique: Saint-Andoche lósz, Delle lwê̌jo, Rougemont $l \bar{u} z \bar{z}$.

$m \ddot{g} g \grave{e}$ (f.) sorte de trempette: Larret $m \grave{g} g \grave{e}$.

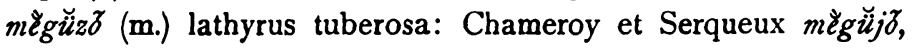
Voisey $m$ ह̌ğ̆zz.

$n \check{e} k$ (f.) roupie (du nez): Saint-Andoche et Chameroy $n \check{k} k$,

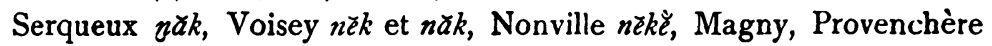
$n \dddot{\imath} k$, Delle $n \check{\imath} t y$, Rougemont $n \check{e} k$, Beauquier naque et niaque.

$n$ Igă̌lărot, fém. pl., (faire à quelqu'un ses -) petits caprices: Voisey faire des nigzlot faire des façons agréables pour enjôler quelqu'un. Probablement parent de fr. mignoter.

păyĕs (f.) bille à jouer: Larret băyĕs, Froideconche payistr, Rougemont balistr, Beauquier baillistes ou -stres, paillistres ou paliestres. Empruntés de ballista.

$p \grave{e}$ (f.) paleron de porc: Chameroy, Serqueux et Voisey pèे.

$\dagger p n \check{a} w(\mathrm{~m})=$.2 cartes: Serqueux pnó, Voisey pnăl pluriel pnó (=1/2 journal), environs de Rougemont pnó; Godefroy penal mesure.

prwéfdü (m.) houx: Provenchère et Val d'Ajol piffó, Plancher pifeu, Delle piffó.

räkně haleter avec bruit, râler: Chameroy räst, Serqueux räsne,

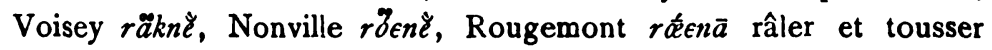
avec difficulté, Plancher rangoina, Beauquier rancoiller, rancot (râle); Godefroy ro onchier ronfler, niçois rangoion râle de l'agonie (v. Calvino Nouv. Dict. nigois-frangais).

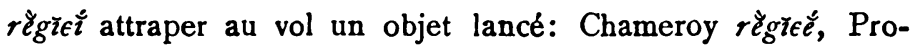
venchère r $r g g_{\epsilon} \in \hat{z}$, Beauquier ragaucher.

rě̀vātť̀ rabrouer, déranger: Chameroy rèveâtě bousculer, déranger, Voisey răvātě̀ dire des choses désagréables.

rêtrǐ̀ très ridé: id. à Voisey, Provenchère, Menoux, Delle.

rgărlǐ très ridé, froncé: Chameroy grălĭ froncé, Serqueux gărlă (se dit surtout d'un fruit que le dessèchement a ridé), Voisey găr grillé (se dit d'une friture, de la viande), Delle grèlè. Ces formes grel-, grŭl ou par métathèse ğ̛̣rl-peuvent dériver de grêle. 
rjăwwpĭ rebondir: Menoux, Provenchère, Froideconche et Rougemont $r z \hat{t} p \vec{a}$.

rkrăple cracher ce qu'on a mis dans sa bouche (non la salive):

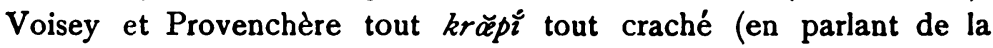
ressemblance), Val d'Ajol rekăăpa, Plancher ekeupa, Rougemont rếkăpāa.

rốfí remuer de place: Voisey rófí (p. ex. le pain dans le four), Delle $r \bar{a} d j \bar{j}$, Rougemont $r \bar{u} d j \bar{i}$.

$r \dot{\alpha}$ (f.) mine menaçante: Nonville rāy, Voisey rákt celui qui fait une mine menaçante (= Pierrecourt rót

sărgăw (m.) cahot: Chameroy, Voisey, Nonville, Val d'Ajol sărgö, Serqueux, Magny, Menoux sěrgö, Provenchère sărğ̋, Froideconche et Delle sërg̈̈, Beauquier sargot.

$s ð r \delta(\mathrm{m}$.) ciron: Provenchère swër $\delta$, Froideconche et Rougemont swar $z$, Plancher çoron. Toutes ces formes correspondent au fr. ciron.

sẼmlé ( $\mathrm{m}$. , non fém.) personne lourde, qui a peine à se remuer: Chameroy sâmtế se dit d'un enfant ou gosse qui tombe à tout obstacle, Voisey samtě.

tăǩֻ compact comme un tissu (se dit du pain), Chameroy tăke,

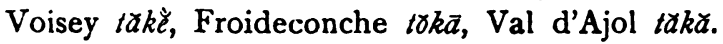

$t \dot{s}$ (f.) très grand tas de gerbes, de foin: Chameroy tis, Serqueux tîs, Provenchère tă $\epsilon$, Menoux tăs, Plancher teuche.

tì $(\mathrm{m}$.) salamandre: Chameroy, Voisey, Rougemont $t ⿱ t$, , Beauquier têt.

Irăk (m.) maladie des cochons (goutte): id. d Serqueux, Menoux, Provenchere.

tră $j$ (m.) passage entre deux maisons: Provenchère, Froideconche trèj; Rougemont trèdj, Beauquier trage ou traige.

văzewsĭ trempé et sali: Provenchère gōsí, Menoux et Froideconche vōsî́, Plancher vauci (salir), Chatenois s vâssîe se crotter.

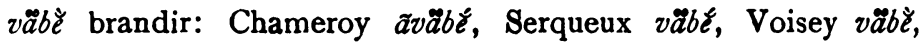
Menoux văba se dit des bras ballants, Beauquier vamber (osciller). Voir Meyer-Lübke, Roman. etym. Wb. art. bambal.

vyōnĕ faire entendre un bruit sifflant: Chameroy vyōné faire une musique désagréable, Voisey vyōně,, Menoux vyōnā (mauvaise musique), Rougemont $v y \bar{u} n \bar{a}$, dolois vionner bruire (toupie qui tourne, v. Ch. Lecomte Le parler dolois). 


\section{Mots patois omis dans le glossaire.}

Il s'agit seulement de mots qui n'ont pas en patois la même prononciation qu'en français régional. Les mots qui se prononcent en patois comme en français régional feront l'objet d'une autre publication dont tous les matériaux sont déjà rassemblés, et où nous aurons à étudier la question de l'emprunt en patois.

$a b z t a \bar{s} y z:(<)$ habitation.

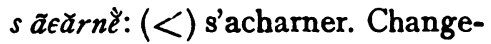
ment de préverbe.

$\tilde{a} v^{\iota}$ (adv.): faire qq. ch. $\tilde{a} v \iota^{\circ}$ malgré soi. Seul emploi. $<$ invitum.

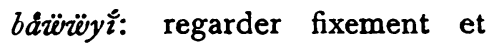
bêtement avec de gros yeux. Chatenois beû̂llîe regarder fixement; Beauquier rebeuiller.

bürtz (m.): petite bure, où l'on met ordinairement de l'huile. Diminutif de burette.

büvrdürüy: $(<)$ bouvreuil. bzāyyt: verbe intransitif qui se dit des vaches qui, piquées des mouches, se sauvent en dressant la queue.

dèbalť: $(<)$ déballer. dŭrzyz: (二) durillon, endroit dur d'une étoffe.

¿gvdy (f.): balayure. Ce qui confirme l'étymologie proposée au Glossaire pour ěgvèyż, dont हैg est le substantif verbal. Chatenois îetyeville balayure du plancher.

ěkdz̈̈r̈̈y: (<) accueil.

ěkrêzdz̈̈rül (f.): personne chétive, enfant chétif. Chatenois îe- crignole (adj.) de chétive apparence.

ëprüvě: (<) approuver.

èr: arrhes.

ĕrăws (f.): $(\Longrightarrow$ arroche.

èstāt tú: (<) statue.

¿̌vnã, -äat: (<) avenant, -ante.

¿kăpāby: incapable.

fyātrí: (二) flétrir.

$f m \bar{c} r$ : fumée. Godefroy $\Longrightarrow$ fumière.

grz̈mönt: grommeler.

jwětyür: $(<)$ jointure.

kăläjł: corriger en fouettant. Emprunté d'une forme méridionale correspondant à v. fr. chalengier.

kavăyt: $(<)$ cavalier. Surtout au bal.

kềbil ( $\epsilon \dot{u}-):(<)$ chou cabus. $k$ bby: $(<)$ comble.

krăsŭ: crasseux. Mais krăsá avare.

$k$ ăvăw (m.): chaufferette.-Dérivé de couver.

küpáby: $(<)$ : coupable.

måēur: $(<)$ mâchoire.

mültz: $(<)$ molleton.

orā̄ty: (<) oracle, c-à-d. homme qui a du babil. 
prăıtè: $(\Longrightarrow$ prêter. Composé: rkdürüy: $(<)$ recueil.

\section{aprăt}

răbo̊ $(p \tilde{o} m-)$ : pomme $(<)$ rambour. rdëvǐr í: retourner (actif). Composé de virit.

rĕ̀vūrtín (f.): quantité trop grande qui est à charge. Ex.: în rĕ̀vūrot $d$ afã. sěvz: $(<)$ savon.

smūy: (>) semoule.

solvåby: $(<)$ solvable.

trẽ: au masc. pl. signifie des linges

de lessive.

\section{Autres additions et corrections.}

Au lieu du signe de la correspondance $\Leftrightarrow$ il faut mettre le signe de l'emprunt $(<)$ aux articles relatifs aux mots suivants: $\bar{a} j$

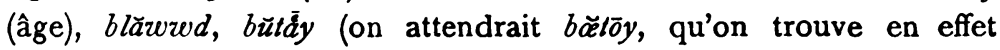

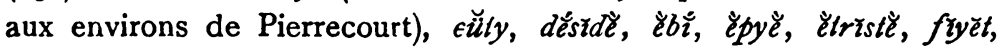

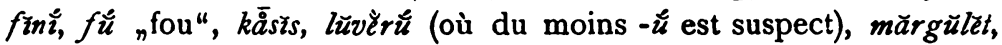

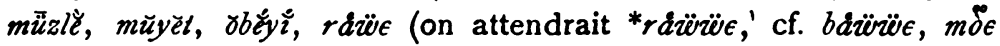

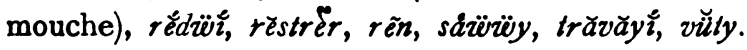

A cause de $a$ au lieu de $\ddot{u}$, sont empruntés à un dialecte

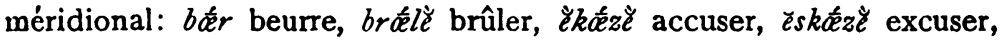

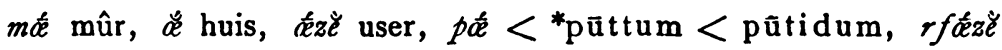
refuser, sátš sucer. Sauf $\ddot{a}$ huis, tous ont un $\not{e}$ long.

$s \bar{q} l$ seigle est sans doute emprunté à un dialecte du nord.

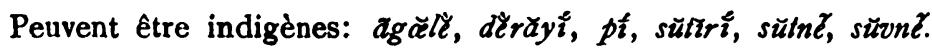

p. 5, lignes 7 et 6 depuis le bas. M. Bertoni me prie d'in-

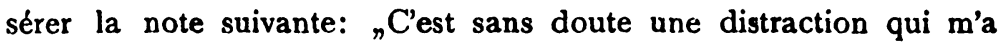
fait dire qu'il existe dans la France du sud toute une aire où - ellum a été supplanté par -ittum. Il existe en effet une aire où - ellum aboutit à -et, mais il s'agit exclusivement d'une règle phonétique (en gascon et béarnais) $-11>t$ ). Je pense pourtant que dans quelques mots -ittum a pu remplacer -ellum."

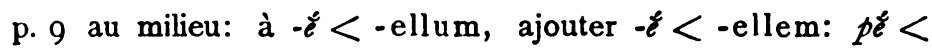
pellem peau.

p. 12, ligne 2 depuis le bas, lire: $\epsilon \breve{u} t y<$ chute, vǚlty $<$ huit.

p. 14, ligne 1, lire: $-\bar{u} r<$-oria ou $-\bar{a}$ tōria.

p. 27, ligne 17 depuis le bas: $s \bar{u} d r$ peut être emprunté au français. 
p. 38 , lignes 4 et suiv. depuis le bas, biffer la remarque I: le cas envisagé est identique au cas cité plus haut, à la même

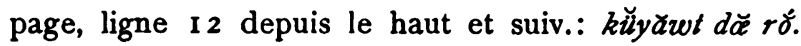
fois sơji.

p. 44, ligne II, ajouter: de même eốjĭ choisir devient par-

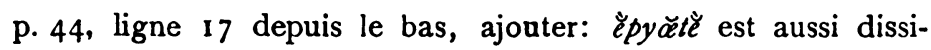

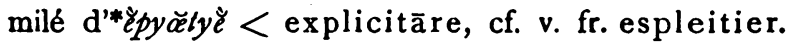

art. apắtürrĭ: ce mot est dérivé de en + pature entrave.

art. äprätĕ: ce mot est non un correspondant de fr. emprunter, mais un composé de prä̌ť̆ prêter.

art. avirzyĩ: lire: donner le vertige.

art. bãbarww: ce mot semble apparenté à prov. babau, v. MeyerLübke, Roman. elym. Wb., art. bab.

art. bä̈̈k: comparer bouquet dartre.

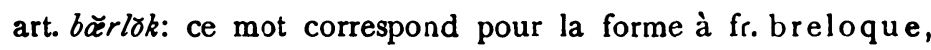
mais pour le sens à patraque, car il désigne tout instrument mal fait, inutilisable, même de grandes dimensions.

art. brd: ce mot évidemment ne peut continuer *berciarium, mais bien v. fr. bers, où -ers est devenu $r d$ comme persica devient prō $\epsilon$.

art. $\epsilon a \ddot{a}:$ lire: cadula, et non caduca. $\epsilon \ddot{a}$ désigne surtout des rillons de porc.

art. $\epsilon \check{u} \mathbf{u} g r \bar{a}:$ lire: rumex.

art. $d z_{z}$, ligne 3 , lire: ${ }^{*} a d b_{\mathfrak{z}} z$.

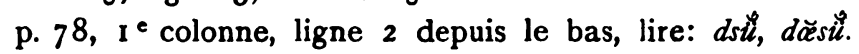

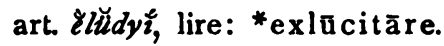

art. ¿mŭyĩ: cf. Chatenois (Vautherin) aimouëillie, en parlant des vaches, annoncer une prochaine parturition par le gonflement mouillé de la vulve; en parler dolois ameiller se dit d'une vache sur le point de vêler (Le parler dolois par Ch. Lecomte 1910).

art. èrğazw: ce mot correspond à fr. ergot.

art. èvezz: ce mot peut être une déformation d'ěllezz, que j’ai trouvé à Larret avec le même sens; ce mot désigne à Pierrecourt et à Larret toute espèce de jalon; êlé̉z peut donc être dérivé d'aligner. Il semble que le -v- de Pierrecourt soit dû à un croisement avec un autre mot, peut-être avec vion qui à Montbéliard signifie jalon (v. Patois de Chatenois, art. vion).

art. fyātrz: ce mot semble dissimilé de *fyätrz, dérivé de fiente, cfr. Fouvent $<$ Fonvans. 
art. füsé: à cause de v. fr. fuissel, il faut partir de *fū scellum, dérivé de fūsum, v. Romania XXVII, 186.

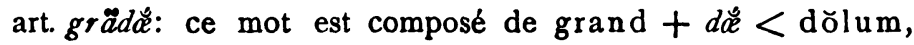
v. fr. doel deuil.

art. grảa (fém.): ce mot continue gratīcla, non -clum.

art. gręv: ce mot désigne non seulement le bas de la jambe, mais surtout la saillie que fait le tibia à la partie ạntérieure de la jambe; il est identique à v. fr. grève; sur l'étymologie de ce mot v. A. Thomas, Romania IyI3.

art. kåz̈̈ souche, cf. bas-limousin couse "cep, pied de vigne" Mais comme cōdicem donnerait *kä̈̈j; il vaut mieux partir de cōdica, dérivé de cōdicem, qui dans un autre sens a donné

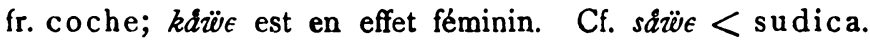

art. kôtz: ce mot est dérivé de fr. côte, même sens.

art. kắtê: au sens de ,heurter de la corne", ce mot est le correspondant de fr. cotir (cf. cosser).

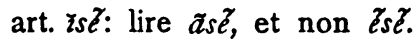

art. māvã: ce mot est une haplologie pour mauvais vent, qu'on emploie aussi dans le même sens.

art. mégè: lire: lat. gaul. *misgo.

art. $m \bar{u} r z$ : ce mot est dérivé de lat. matūrum, cf. mavurons (même sens) chez Beauquier Vocabulaire elym. des provincialismes du Doubs.

art. $\delta d 8$ : ce mot est dérivé de ordinem, v. Meyer-Lübke, Roman. elym. Wb. art. ordināre.

art. $\delta 8 \check{a} r Z_{n}$ : cette forme est un barbarisme; la forme authentique

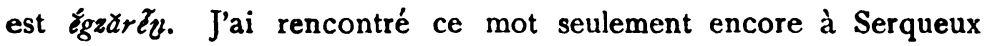
(Haute-Marne), où l'on prononce ègex̌rin.

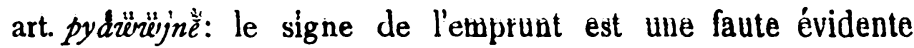
d'impression; pjäz̈rïjjnĕ correspond à l'ital. piovigginare, mais n'en vient pas.

art. rjêzzé: v. Roman. elym. Wb. art. ingannāre.

art. rnăzęl grenouille rainette: sur d'autres formes de ce mot v. A. Thomas, Melanges p. 125-6.

art. rấ: ajouter: ce mot désigne aussi les raies ou sillons qu'on forme en mettant le fourrage en raie dans un pré en fanant. En ce sens il peut être identique d̀ rá mine menaçante (v. Glossaire $d u$ patois de Pierrecourt); on peut l'expliquer par lat. rūga ride, fronce: faire la $r^{\mathfrak{a}}$ serait en cette hypothèse "faire la fronce", 
184 C. JURET, QURIQUES ADDITIONS AU GLOSSAIRE DE PIERRECOURT.

froncer les sourcils d'un air menaçant. En niçois rua signifie ride et fronce (v. Calvino Nouveau dict. nicois-francais). Dans le „Parler dolois" (arr. de S. Malo) par $\mathrm{Ch}$. Lecomte, je relève aussi: faire la reu regarder de travers (p. 228).

rtrātyăť̌ ou trătyăť̆ ne signifie pas "refaire une besogne" quelconque, mais raccommoder vaille que vaille, surtout réparer une chaîne cassée, en l'absence de tout ouvrier compétent, avec des moyens de fortune.

C. JURET. 